taining, expanding, and solving the secular determinants) were carried out numerically.

The force constants given in Table VII were evaluated from known frequencies of ethane, propane, as shown in the table. The frequencies calculated from these force constants, which are discussed in Sections III and IV are:

$\begin{array}{lll}\text { Isobutane: } & A_{1} & 776 \mathrm{~cm}^{-1} \\ & & (434) \\ & E & 1034 \\ & & 369 \\ \text { Neopentane: } & A_{1} & 729 \mathrm{~cm}^{-1} \\ & E & 230 \\ & T_{2} & 983 \\ & 422\end{array}$

\section{APPENDIX B}

\section{HCC Bending Force Constant Calculations on Propane and Isobutane}

In the models used, the $\mathrm{CH}_{3}$ groups were considered as point masses, but the secondary and tertiary hydrogens were included in the calculations. The methods used were the same as those described in Appendix A, with the additional simplification that the $\mathrm{CH}$ stretching force constants were set equal to infinity and the $\mathrm{CH}$ stretching vibrations were separated from the others and subsequently ignored, according to the procedure given by Wilson. ${ }^{7}$

In order to obtain suitable force constants for use with these models, calculations were made under similar assumptions on methane and ethane. The force constants used, and their sources, are given in Table VIII. The calculated frequencies, which are discussed in Sections III and IV, are:

$\begin{array}{lcc}\text { Propane: } & A_{1} & 898 \mathrm{~cm}^{-1}(\mathrm{C}-\mathrm{C} \text { stretching) } \\ & & 1467(\mathrm{HCH} \text { bending) } \\ & 427(\mathrm{C}-\mathrm{C}-\mathrm{C} \text { bending }) \\ & A_{2} & 1175(\mathrm{CH} \text { twisting }) \\ & B_{1} & 998(\mathrm{C}-\mathrm{C} \text { stretching }) \\ & & 1205\left(\mathrm{CH}_{2} \text { wagging }\right) \\ & B_{2} & 761\left(\mathrm{CH}_{2} \text { rocking) }\right. \\ \text { Isobutane: } & A_{1} & 821 \mathrm{~cm}^{-1}(\mathrm{C}-\mathrm{C} \text { stretching }) \\ & & 571(\mathrm{C}-\mathrm{C}-\mathrm{C} \text { bending }) \\ & E & 994(\mathrm{C}-\mathrm{C} \text { stretching }) \\ & 319(\mathrm{C}-\mathrm{C}-\mathrm{C} \text { bending }) \\ & & 1113(\mathrm{CH} \text { wagging })\end{array}$

\title{
The Nature of Bond Orbitals in Quadricovalent Complexes of Transition Elements*
}

\author{
HANS KuHN** \\ Gates and Crellin Laboratories, California Institute of Technology, Pasadena, California
}

(Received April 7, 1948)

\begin{abstract}
In quadricovalent complexes of transition elements ( $\mathrm{Ni}^{\mathrm{II}}, \mathrm{Pd}^{\mathrm{II}}, \mathrm{Pt}^{\mathrm{II}}, \mathrm{Au}^{\mathrm{III}}, \mathrm{Ag}^{\mathrm{II}}, \mathrm{Cu}{ }^{\mathrm{II}}$ ) the central atom uses four $s$ or $d$ orbitals of its outer shell for unshared electron pairs and two $s$ or $d$ and two $p$ orbitals for bond formation. The problem here is to find the strongest possible four hybrid bond orbitals obtained by combining the two $s-d$ and the two $p$ orbitals available for bond formation. The strongest bond orbitals thus obtained are directed from the middle towards the four corners of a square, as it is expected from experimental evidence; they are composed of $4 / 9 s, 14 / 9 d$, and two $p$ orbitals, the strength being 2.943. These strongest possible orbitals are presumably used in complex of $\mathrm{Ni}^{\mathrm{II}}, \mathrm{Pt}^{\mathrm{II}}, \mathrm{Au}^{\mathrm{III}}$, and $\mathrm{Cu}^{\mathrm{II}}$, but probably not in complexes of $\mathrm{Pd}^{\mathrm{II}}$ and $\mathrm{Ag}^{\mathrm{II}}$; in the latter two cases there must be assumed square bond orbitals of composition $s^{n} d^{2-n} p^{2}$ with $n$ somewhere between 1 and $4 / 9$, i.e., orbitals of character between that of the strongest square bond orbitals of composition $s^{4 / 9} d^{14 / 9} p^{2}$ mentioned above and that of $s d p^{2}$ square orbitals discovered by Pauling.
\end{abstract}

\section{INTRODUCTION}

$T$ HE problem of directed valence was first developed by Pauling ${ }^{1}$ and Slater. ${ }^{2}$ Pauling

\footnotetext{
* Contribution No. 1187 from Gates and Crellin Laboratories.

** Present address: Institute of Physical Chemistry of the University of Basel, Basel, Switzerland.

1 L. Pauling, J. Am. Chem. Soc. 53, 1367 (1931); The Nature of the Chemical Bond (Cornell University Press, Ithaca, New York, 1945), second edition.

2 J. C. Slater, Phys. Rev. 37, 481 (1931).
}

formulated a very simple method of discussing the bond-forming power of an atomic bond orbital. The fundamental assumption of this method is that in the case of bond orbitals with the same or nearly the same radial part of the wave function the magnitude of the angular part of a bond along the bond axis (that is, in the direction of the ligand) is a measure of its relative bond-forming power. 


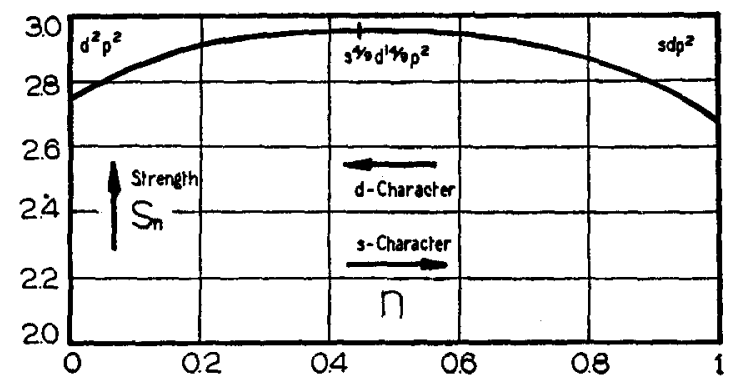

FIG. 1. Square bond orbitals. Plot of the strength, $S_{n}$, against the amount, $n$, of $s$ orbitals involved. As there are exactly two $p$ orbitals involved in each square arrangement, the amount of $d$ orbitals is $2-n$, the arrangement thus being $s^{n} d^{2-n} p^{2}$.

The bond-forming power or strength $S$ of an $s$ orbital defined in this way is 1 , that of a $p$ orbital $\sqrt{3}$, and that of the best $d$ orbital $(5)^{\frac{1}{2}}$.

Pauling has shown that it is possible, by combining $s$ and $p$ or $s, p$, and $d$ orbitals, to obtain a number of strong bond orbitals (i.e., orbitals with large $S$ ). For example, he has found combinations of $s$ and $p$ orbitals which are directed towards the corners of a tetrahedron, combinations of $s, p$, and $d$ orbitals which are directed toward the corners of a square, and other combinations which are directed towards the corners of an octahedron.

An important result of Pauling's approach is that the four $s p$ orbitals in the directions of the corners of a regular tetrahedron are the strongest possible combinations of $s$ and $p$ orbitals. Thus the tetrahedral valence of carbon and similar elements has found a simple explanation.

In contrast to carbon are the $B$ group elements such as $\mathrm{Ni}^{\mathrm{II}}, \mathrm{Pd}^{\mathrm{II}}, \mathrm{Pt}^{\mathrm{II}}, \mathrm{Cu}^{\mathrm{II}}$, and $\mathrm{Au}^{\mathrm{III}}$, surrounded by four electronegative atoms generally in a square, and not tetrahedral, arrangement. Pauling has shown that four rather strong bond orbitals with strength $S=2.694$ can be constructed for this square arrangement by hybridization of one $s$, one $d$, and two $p$ orbitals.

The question as to whether or not the square bond orbitals discussed by Pauling are the strongest possible four hybrid orbitals has not before been answered. We may think of the possibility of constructing four stronger $s p d$ orbitals than the square orbitals; it must be decided whether or not the concept of bond strength enables us to exclude other arrangements as less stable, such as a tetrahedral or a tetragonal pyramidal arrangement.

\section{ELECTRONIC STRUCTURE AND BOND AR- RANGEMENT OF QUADRICOVALENT COM- PLEXES OF TRANSITION ELEMENTS}

\section{a. Square Complexes of $\mathrm{Ni}^{\mathrm{II}}, \mathrm{Pt}^{\mathrm{II}}$, and $\mathrm{Au}^{\mathrm{III}}$}

Let us look at a covalent complex of bivalent nickel, such as the nickel cyanide ion $\left[\mathrm{Ni}(\mathrm{CN})_{4}\right]^{--}$. The twenty-six electrons of $\mathrm{Ni}^{\mathrm{II}}$ can be placed in the following way: 18 electrons can be placed in the $1 s, 2 s$, three $2 p, 3 s$, and three $3 p$ orbitals; for the other eight electrons six orbitals, namely, the five $3 d$ orbitals and the almost equally stable $4 s$ orbital, are available, and four of these orbitals may be occupied by electron pairs. ${ }^{3}$

This leaves available for use in bond formation two orbitals of the $3 d-4 s$ shell as well as the three $4 p$ orbitals, which are somewhat less stable than the $3 d$ and $4 s$ orbitals., 5 As four bonds are to be formed, the smallest possible amount of unstable $4 p$ orbitals, namely two, should be used. We are left with the problem of determining the geometric arrangement of the four strongest $s p d$ hybrid orbitals that can be formed from two $p$ and two $s-d$ orbitals and that are, at the same time, orthogonal to four orbitals with pure $4 s$ and $3 d$ character (these orbitals being filled with eight electrons of the nickel shell) and to a pure $p$ orbital (this orbital being empty).

For this purpose we shall discuss several geometrical arrangements of four $s p d$ bond orbitals, and we shall find that of all arrangements of equivalent orbitals only square arrangements have, in fact, the property stated above of being orthogonal to four $s-d$ orbitals and one $p$ orbital. In Fig. 1 the strength, $S_{n}$, of these orbitals as ordinate is plotted against the amount, $n$, of involved $s$ orbital as abscissa. The square orbitals

3 The nearly equal stability of $3 d$ and $4 s$ follows from the position of atomic spectral terms in nickel. The ground state of nickel is a ${ }^{3} \mathrm{~F}$ state corresponding to a configuration $3 d^{8} 4 s^{2}$; the lowest excited state ${ }^{3} \mathrm{D}$, corresponding to a structure $3 d^{9} 4 s$, is only $0.58 \mathrm{kcal}$./mole above the ground state (see reference 4 ). As this energy difference is small compared to any covalent bond energy, it can be neglected in our treatment and $4 s$ and $3 d$ can be regarded as equally stable.

4 The difference in stability of $4 s$ and $4 p$ orbitals may be seen from the spectral terms of nickel. The ${ }^{3} P^{\circ}$ term corresponding to the electronic structure $3 d^{9} 4 p$ is $81 \mathrm{kcal} . / \mathrm{mole}$ above the state $3 d^{9} 4 s^{3} D$ (see reference 5).

${ }^{5}$ R. F. Bacher and S. Goudsmit, Atomic Energy States (McGraw-Hill Book Company, New York, 1932). 
that must be assumed to be actually used in covalent complexes of bivalent nickel are the orbitals with maximum strength. These orbitals are composed of $0.44 s, 1.56 d$, and $2.00 p$ orbitals, the strength of each orbital being 2.943 .

These strongest square orbitals are presumably also used in covalent complexes of $\mathrm{Pt}^{\mathrm{II}}$ and $A u^{I I I}$. There is the same number of electrons involved in the outmost $5 d-6 s$ shell of $\mathrm{Pt}^{\mathrm{II}}$ and $A u^{I I I}$ as in the $3 d-4 s$ shell of $\mathrm{Ni}^{\mathrm{II}}$ and furthermore it can be assumed ${ }^{6}$ that the $5 d$ and $6 s$ orbitals are nearly equal in stability, as are $3 d$ and $4 s$ in nickel; therefore, the same discussion is applicable.

\section{b. Complexes of $\mathbf{P d}^{\mathrm{II}}$}

A somewhat different case is found in square complexes of $\mathrm{Pd}^{\mathrm{II}}$. The $4 d$ and $5 s$ orbitals in $\mathrm{Pd}$ cannot be assumed as equally stable. If we place 36 of the 44 electrons of $\mathrm{Pd}^{\text {II }}$ in the inner shells and the other 8 electrons in four of the five $4 d$ orbitals, we obtain a state which is, by about 50 $\mathrm{kcal} . /$ mole, more stable than the state containing the four electron pairs in the $5 s$ and in three of the five $4 d$ orbitals. ${ }^{7}$ In bond formation, hence, there will be a tendency to use as much of the stable $4 d$ orbitals as possible for unshared pairs, leaving the less stable $5 s$ and $5 p$ as well as one of the $4 d$ orbitals for bond formation; this tendency by itself would lead to the $s p^{2} d$ square arrangement found by Pauling. ${ }^{1}$ On the other hand, there will be, as in the cases discussed above, a tendency to use the strongest possible bond orbitals, i.e., the $s^{0.44} d^{1.56} p^{2}$ square bond orbitals. In consequence a compromise will be reached; the bond orbitals will utilize less than

\footnotetext{
${ }^{6}$ From the atomic spectra of platinum it can be seen (see reference 4 ) that the excited state $5 d^{9} 6 s^{3} D$ is only $2.35 \mathrm{kcal}$./mole above the ground state $5 d^{8} 6 s^{2}{ }^{3} F$.

The difference in stability between $6 s$ and $6 p$ orbitals in $\mathrm{Pt}$ may be of interest. The state $5 d^{9} 6 p^{3} P$ is not known in platinum, but it can be estimated that this state is, as in the case of nickel, about $80-90 \mathrm{kcal}$./mole less stable than the state $5 d^{9} 6 s^{3} \mathrm{D}$. (This is seen in the following way: the energy difference between the $d^{9} s^{3} D$ and the $d^{3} p^{3} P$ states in the sequence $\mathrm{Ni}, \mathrm{Cu}^{+1}, \mathrm{Zn}^{+2}$ are $81,126,170$ $\mathrm{kcal} . / \mathrm{mole}$, respectively; the corresponding energy values for $\mathrm{Au}^{+1}$ and $\mathrm{Hg}^{+2}$ are 137 and $173 \mathrm{kcal}$./mole, respectively (see reference 4 ); the value in the case of $\mathrm{Pt}$, the element next to the left of Au in the periodic table, is thus found by extrapolation to be about $90 \mathrm{kcal} . / \mathrm{mole}$.)

7 This can be concluded from the atomic spectral terms of Pd. The energy difference between the state $4 d^{9} 5 s^{3} D$ and the higher state $4 d^{8} 5 s^{2} 3 \mathrm{~F}$ is $52.8 \mathrm{kcal} . / \mathrm{mole}$ in $\mathrm{Pd}$. (See reference 4.)
}

one and more than $0.44 s$ orbital; we will thus have $s^{n} d^{2-n} p^{2}$ square bond orbitals with $n$ somewhere between 1 and 0.44 (see Fig. 1).

The value of $n$ in a given case can be found by the following treatment:

We shall first assume that $s p^{2} d$ square orbitals are used for bond formation, then we shall replace these orbitals by $s^{n} p^{2} d^{2-n}$ bond orbitals, $n$ being arbitrary within the limits 0 and 1. The energy difference which is involved in this replacement produces the sum of two parts.

On one hand, the strength of the bond orbitals and therefore the covalent bond energy is changed. Let us denote by $W_{n=1}$ the energy of a bond between the central atom and one of its ligands in the case where the $s p^{2} d$ square orbitals of strength $S_{n \rightarrow 1}=2.694$ are used in bond formation. The bond energy that has to be assumed in case that the central atom uses $s^{n} p^{2} d^{2-n}$ bond orbitals of strength $S_{n}$ is then equal to $W_{n}=W_{n=1} \cdot\left(S_{n}^{2} / S_{n=1}{ }^{2}\right)$. This follows from a theorem by Pauling, according to which the energy of a bond is proportional to the product of the squares of the strengths of the orbitals of the two atoms involved. Hence, the change of bond energy in the replacement that we are considering (formation of four bonds involving $s^{n} p^{2} d^{2-n}$ square orbitals instead of bonds involving $s p^{2} d$ orbitals $)$ is equal to $4 W_{n=1}\left(\left(S_{n}^{2} / S_{n=1}^{2}\right)-1\right)$.

On the other hand, the electronic energy is changed in the replacement under consideration. At first, when $s p^{2} d$ orbitals are used in bond formation no unstable $s$ orbital. is used for unshared pairs. However, when $s^{n} p^{2} d^{2-n}$ bond orbitals are used, an amount $(1-n)$ of an $s$ orbital is needed for unshared pairs. The interaction energy of a shared pair is in the case of a pure covalent bond the same as that of a single unshared electron; ${ }^{1}$ therefore, the energy required for the interchange of $(1-n)$ shared pairs and $(1-n)$ unshared pairs between $s$ and $d$ orbitals is equal to $(1-n) \Delta E, \Delta E$ being the $s-d$ separation energy, i.e., the energy required for the transport of one electron from the $d$ into the less stable $s$ orbital. Assuming, however, that there are not pure covalent bonds between the central atom and its ligands but bonds with, say, 100- $P$ percent ionic character (the attached atoms being more electronegative than the central atom), the interaction energy of a shared pair with the central atom is equal to that of only $P$ percent of a single electron. The energy required for the interchange of a shared and an unshared pair between $s$ and $d$ orbitals is thus equal to $(2-P / 100) \Delta E$ and the energy required for interchanging $(1-n)$ shared and unshared pairs equal to $(1-n)(2-P / 100) \Delta E$.

Therefore, the total energy increase $\Delta U$ in the replacement under consideration is given by

$$
\Delta U=-4 W_{n=1}\left(\left(S_{n}^{2} / S_{n=0}{ }^{2}\right)-1\right)+\Delta E(1-n)(2-P / 100) \text {. }
$$

The $n$ value actually used in bond orbital formation is obtained by minimizing $\Delta U$ in respect to $n$. We obtain:

$$
(2-P / 100)\left(\Delta E / W_{n=1}\right)=-\left(8 / S_{n=0}^{2}\right) S_{n}\left(d S_{n} / d n\right) .
$$

By substituting $S_{n}$ from Eqs. (10) and (10a) mentioned later we obtain the following values of $n$ for various values 


of $\begin{array}{cc}\Delta E / W_{n=1} \times(2-P / 100): & \\ \left(\Delta E / W_{n=1}\right)(2-P / 100) & n \\ 0.0 & 4 / 9 \\ 0.27 & 5 / 9 \\ 0.58 & 6 / 9 \\ 1.0 & 7 / 9 \\ 1.8 & 8 / 9 \\ \infty & 1\end{array}$

The bond energy of a single bond in complexes of transition elements may be of the order of 60 to $100 \mathrm{kcal} . / \mathrm{mole}$, and, according to Pauling, ${ }^{8}$ these bonds are in most cases about 50 percent covalent in character, $P$ thus being about equal to 50 ; furthermore, the $s-d$ separation energy $\Delta E$ in the case of palladium is $50 \mathrm{kcal} . / \mathrm{mole}$, as has been mentioned above; the expression $\Delta E / W_{n=1}(2-P / 100)$ hence will be between 0.7 and 1.4 ; therefore, $n$ probably lies between $2 / 3$ and $8 / 9$ in palladium complexes.

\section{c. Complexes of $\mathrm{Cu}^{\mathrm{II}}$ and $\mathbf{A g}^{\mathrm{II}}$}

In bivalent copper conditions are similar to those in bivalent nickel. Bivalent copper has, however, one electron more than bivalent nickel; we might.at first think that this electron would occupy the fifth $3 d$ or the about equally stable $4 s$ orbital, making it unavailable for bond formation; this would leave available one $3 d-4 s$ and three $4 p$ orbitals for bond formation. However, as has been pointed out by Pauling, ${ }^{1}$ placing the unshared electron in the third $4 p$ orbital (or in a $4 s 4 p 3 d$ hybrid orbital) and using the residual four orbitals for bond formation involves no loss of energy by the copper atom. Each of the five orbitals under discussion (two $4 s-3 d$ orbitals and three $4 p$ orbitals) is occupied either by a shared pair or by the single unshared electron in either formulation, and the interaction energy of a shared pair with the copper atom is the same as that of a single unshared electron. ${ }^{9}$

${ }^{8} \mathrm{~L}$. Pauling, The Valences of Transition Elements (Victor Henri Memorial Volume, Liège, 1947).

${ }^{9}$ In Section $2 \mathrm{~b}$ we have seen that the interaction energy of a shared pair with the central atom is only in the case of pure covalent bonds equal to that of a single unshared electron, and that these energies are somewhat different in the case of covalent bonds partially ionic in character. A consideration similar to that given in Section $2 \mathrm{~b}$, the details of which are omitted, has shown that the difference of these two interaction energies is in any reasonable case too small for effecting a change of the nature of the most stable bond orbitals, and shall be neglected here for reason of simplicity.
These considerations leave us with the problem of determining the geometric arrangement of the four strongest $s p d$ hybrid orbitals that are orthogonal to four orbitals with pure $s-d$ character (these orbitals being filled with unshared pairs) and to an orbital of arbitrary composition in $s, p$, and $d$ (this orbital being filled with the single unshared electron).

We shall find in the following treatment that the best possible orbitals that can be constructed under this condition are again the best square bond orbitals with strength 2.943 described above. These orbitals thus must be assumed for $\mathrm{Cu}^{\mathrm{II}}$ complexes, which are in fact found to have a square arrangement.

Quadricovalent complexes of bivalent silver, like those of bivalent copper, are found to be coplanar. The same number of electrons are involved in the outer shell of $\mathrm{Ag}^{\mathrm{II}}$ as in the outer shell of $\mathrm{Cu}^{\mathrm{II}}$, and therefore a similar discussion is applicable. It must be assumed, however, that the $5 s$ orbital in silver, as in palladium, is considerably less stable than the $4 d$ orbitals (while the outer $3 d$ and $4 s$ orbitals in copper, as in nickel, must be assumed about equally stable). Consequently, there will be used in quadricovalent complexes of silver as in complexes of palladium $s^{n} d^{2-n} p^{2}$ square bond orbitals, with $n$ somewhere between 1 and 0.44 , the odd electron of silver being in the third $5 p$ orbital.

\section{THE DIFFERENT POSSIBLE ARRANGEMENTS OF FOUR $s p d$ BOND ORBITALS AND THEIR BOND-FORMING POWER}

It has been shown by Kimball ${ }^{10}$ by group theoretical considerations that it is possible to construct from $s, p$, and $d$ orbitals four equivalent bond orbitals in either a tetragonal plane, a tetragonal pyramidal, a regular tetrahedral, or an irregular (trigonal axis deformed) tetrahedral arrangement, and no other. The geometrical arrangement of the strongest possible equivalent $s p d$ orbitals can thus be found by determining the angular dependence of orbitals in only these four arrangements and by determining which of the four arrangements is the best.

${ }^{10} \mathrm{G}$. Kimball, J. Chem. Phys. 8, 188 (1940). See also R. Hultgren, Phys. Rev. 40, 891 (1932). 


\section{a. Tetragonal Plane Bond Orbital Arrangement}

The angular parts of the $s, p$, and $d$ wave functions are $:^{1}$

$$
\begin{aligned}
s & =1, \\
p_{x} & =\sqrt{3} \sin \vartheta \cos \varphi, \\
p_{y} & =\sqrt{3} \sin \vartheta \sin \varphi, \\
p_{z} & =\sqrt{3} \cos \vartheta \\
d_{x y} & =\left((15)^{\frac{1}{2}} / 2\right) \sin ^{2} \vartheta \cos 2 \varphi, \\
d_{x+y} & =\left((15)^{1 / 2}\right) \sin ^{2} \vartheta \sin 2 \varphi, \\
d_{z} & =\left((5)^{\frac{1}{2}} / 2\right)\left(3 \cos ^{2} \vartheta-1\right), \\
d_{x+z} & =(15)^{\frac{1}{2}} \sin \vartheta \cos \vartheta \cos \varphi, \\
d_{y+z} & =(15)^{\frac{1}{2}} \sin \vartheta \cos \vartheta \sin \varphi,
\end{aligned}
$$

$\vartheta$ and $\varphi$ being the angles used in spherical polar coordinates.

The bond direction of a first square bond orbital will be chosen as the $x$ direction. It is easily seen that inclusion of $p_{y}, p_{z}, d_{x+y}$, or $d_{x+z}$ orbitals would cause the bond direction to be different from the $x$ direction, so they are ignored. It can also be shown that $d_{y+z}$ does not increase the strength of a square orbital in the $x$ direction but decreases it, so it is ignored, too, the function thus assuming the form

$$
\psi_{1}=a_{s} s+a_{p} p_{x}+d_{d x y} d_{x y}+a_{d z} d_{z}
$$

By rotating $\psi_{1}$ around the $z$ axis through angles of rotation of $90^{\circ}, 180^{\circ}$, and $270^{\circ}$, respectively, the other equivalent square bond orbitals are easily found to be

$$
\begin{aligned}
& \psi_{2}=a_{8} s+a_{p} p_{y}-a_{d x y} d_{x y}+a_{d z} d_{z}, \\
& \psi_{3}=a_{s} s-a_{p} p_{x}+a_{d x y} d_{x y}+a_{d z} d_{z}, \\
& \psi_{4}=a_{s} s-a_{p} p_{y}-a_{d x y} d_{x y}+a_{d z} d_{z} .
\end{aligned}
$$

The orthogonality and normalization conditions require that

$$
\begin{aligned}
a_{s}{ }^{2}+a_{p}{ }^{2}+a_{d x y}{ }^{2}+a_{d z}{ }^{2} & =1, \\
a_{s}{ }^{2}-a_{p}{ }^{2}+a_{d x y}{ }^{2}+a_{d z}{ }^{2} & =0, \\
a_{s}{ }^{2}-a_{d x y}{ }^{2}+a_{d z}{ }^{2} & =0 .
\end{aligned}
$$

We obtain from these equations the results

$$
\begin{aligned}
a_{p}{ }^{2} & =\frac{1}{2}, \\
a_{d x y} & =\frac{1}{4}, \\
a_{8}{ }^{2}+a_{d z}{ }^{2} & =\frac{1}{4} .
\end{aligned}
$$

It is seen from (6) that there is always $\frac{1}{2} p$ orbital involved in a square bond orbital and thus exactly two $p$ orbitals in each square arrangement. Furthermore, it follows from (7) that the $d_{x y}$ orbital is used completely in each square arrangement.

We obtain, by introducing (6), (7), and (8) into (2a),

$$
\psi_{1}=a_{s} s+(1 / \sqrt{2}) p_{x}+\frac{1}{2} d_{x y}-\left(\frac{1}{4}-a_{s}^{2}\right)^{\frac{1}{3}} d_{z},
$$

Similar expressions are obtained from $(2 \mathrm{~b}),(2 \mathrm{c})$, and (2d).

The strength $S_{n}$ is easily found from (9) and (1) :

$$
S_{n}=a_{s}+\sqrt{3} / \sqrt{2}+\left((15)^{\frac{1}{1}} / 4\right)+\left((5)^{\frac{1}{3}} / 2\right)\left(\frac{1}{4}-a_{s}^{2}\right)^{\frac{1}{3}} .
$$

The quantity $S_{n}$, as given by Eq. (10), is plotted in Fig. 1 against the amount

$$
n=4 a_{s}^{2}
$$

of involved $s$ orbital.

The value of $a_{8}$ corresponding to the maximum strength is found, by maximization of $(10)$, to be $\frac{1}{3}$, the corresponding strength being $S=2.943$. The angular wave functions of these strongest square bond orbitals are thus found to be

$$
\begin{aligned}
& \psi_{1}=\frac{1}{3} s+(1 / \sqrt{2}) p_{x}+\frac{1}{2} d_{x y}-\left((5)^{\frac{3}{3}} / 6\right) d_{z}, \\
& \psi_{2}=\frac{1}{3} s+(1 / \sqrt{2}) p_{y}-\frac{1}{2} d_{x y}-\left((5)^{\frac{1}{1}} / 6\right) d_{z}, \\
& \psi_{3}=\frac{1}{3} s-(1 / \sqrt{2}) p_{x}+\frac{1}{2} d_{x y}-\left((5)^{\frac{1}{2}} / 6\right) d_{z}, \\
& \psi_{4}=\frac{1}{3} s-(1 / \sqrt{2}) p_{y}-\frac{1}{2} d_{x y}-\left((5)^{\frac{1}{2}} / 6\right) d_{z} .
\end{aligned}
$$

This strongest arrangement of square bond orbitals is thus composed of $4 / 9 s, 14 / 9 d$, and two $p$ orbitals.

It is of interest to determine the nature of the five remaining orbitals, which are orthogonal to the square bond orbitals and are not involved in bonds. There are, of course, the four orbitals $p_{2}$, $d_{x+y}, d_{x+z}$, and $d_{y+z}$ that are not used in hybridization. The fifth is the orbital

$$
\psi_{5}=\left(1-4 a_{8}^{2}\right)^{\frac{1}{s}} s+2 a_{8} d_{z}
$$

which is formed from the parts of $s$ and $d_{z}$ orbitals that are not needed in bond orbital formation. In the case of the strongest square bond orbitals (11a), (11b), (11c), (11d), $\psi_{5}$ is given by

$$
\psi_{5}=\left((5)^{\frac{1}{3}} / 3\right) s+\frac{2}{3} d_{2}=(5)^{\frac{1}{3}} \cos ^{2} \vartheta,
$$


which gives a strength of $(5)^{1}$, that is, just the strength of the best single $d$ orbital. The possibility of using this orbital for a weak bond along the $z$ axis is not excluded.

\section{b. Regular Tetrahedral Bond Orbitals}

We shall now look for spd hybrid orbitals for bonds directed from the central atom to the four corners of a regular tetrahedron. The bond direction of a first hybrid orbital will be chosen as the $z$ direction. As we restrict ourselves for reasons of simplicity to cylindrical orbitals, the orbitals $p_{x}, p_{y}, d_{x y}, d_{x+y}, d_{x+z}$, and $d_{y+z}$ are ignored, and the function assumes the form

$$
\psi_{1}=a_{s} s+a_{p} p_{z}+a_{d} d_{z} .
$$

The other three equivalent tetrahedral bond functions $\psi_{2}, \psi_{3}$, and $\psi_{4}$ are easily found by rotating $\psi_{1}$ first around the $y$ axis through an angle of $109^{\circ} 28^{\prime}$ (tetrahedral angle) and then around the $z$ axis through angles $0^{\circ}, 120^{\circ}$, and $240^{\circ}$, respectively.

$$
\begin{gathered}
\psi_{2}=a_{8} s-\frac{1}{3} a_{p} p_{z}+(2 \sqrt{2} / 3) a_{p} p_{x}-\frac{1}{3} a_{d} d_{z} \\
+(4 / 3 \sqrt{3}) a_{d} d_{x y}-(2 \sqrt{2} / 3 \sqrt{3}) a_{d} d_{x+z}, \\
\begin{aligned}
\psi_{3}=a_{s} s-\frac{1}{3} a_{p} p_{z}- & (\sqrt{2} / 3) a_{p} p_{x}-(\sqrt{2} / \sqrt{3}) a_{p} p_{y} \\
-\frac{1}{3} a_{d} d_{z}- & (2 / 3 \sqrt{3}) a_{d} d_{x y}+(\sqrt{2} / 3 \sqrt{3}) a_{d} d_{x+z} \\
+ & (\sqrt{2} / 3) a_{d} d_{y+z}+\frac{2}{3} a_{d} d_{x+y}
\end{aligned} \\
\begin{aligned}
& \psi_{4}=a_{s} s-\frac{1}{3} a_{p} p_{z}-(\sqrt{2} / 3) a_{p} p_{x}+(\sqrt{2} / \sqrt{3}) a_{p} p_{y} \\
&-\frac{1}{3} a_{d} d_{z}-(2 / 3 \sqrt{3}) a_{d} d_{x y}+(\sqrt{2} / 3 \sqrt{3}) a_{d} d_{x+z} \\
&-(\sqrt{2} / 3) a_{d} d_{y+z}-\frac{2}{3} a_{d} d_{x+y}
\end{aligned}
\end{gathered}
$$

We shall require that these functions be normalized to $4 \pi$ and be mutually orthogonal. These

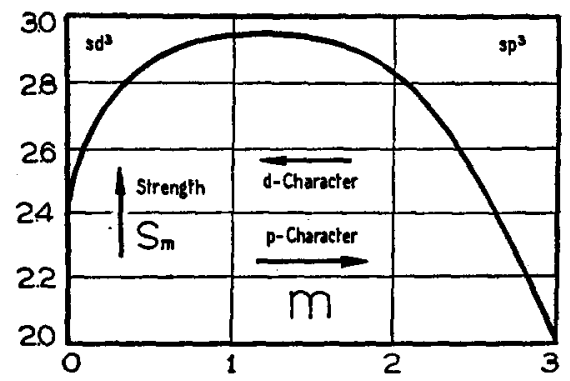

FIG. 2. Regular tetrahedral bond orbitals. Plot of the strength, $S_{m}$, against the amount, $m$, of $p$ orbitals involved. As there is exactly one $s$ orbital involved in each tetrahedral arrangement, the amount of $d$ orbitals is $3-m$, the arrangement thus being $s p^{m} d^{3-m}$. conditions lead to the relations

$$
\begin{aligned}
a_{s}^{2}+a_{p}^{2}+a_{d}^{2} & =1, \\
a_{8}^{2}-\frac{1}{3} a_{p}{ }^{2}-\frac{1}{3} a_{d}^{2} & =0 .
\end{aligned}
$$

We obtain from these equations the result

$$
\begin{aligned}
a_{s}^{2} & =\frac{1}{4}, \\
a_{p}{ }^{2}+a_{d}{ }^{2} & =\frac{3}{4} .
\end{aligned}
$$

It can be seen from (17) that there is always $\frac{1}{4} s$ orbital involved in a tetrahedral orbital and thus exactly one $s$ orbital in each tetrahedral arrangement.

We obtain by introducing (17) and (18) into (14a) the expression

$$
\psi_{1}=\frac{1}{2} s+a_{p} p_{z}+\left(\frac{3}{4}-a_{p}\right)^{\frac{1}{3}} d_{z} .
$$

Similar expressions for $\psi_{2}, \psi_{3}, \psi_{4}$ are obtained from $(14 b),(14 c)$, and (14d). The strength of these orbitals,

$$
S_{m}=\frac{1}{2}+\sqrt{3} a_{p}+(5)^{\frac{1}{2}}\left(\frac{3}{4}-a_{p}^{2}\right)^{1},
$$

is shown in Fig. 2 as a function of the number $m=4 a_{p}{ }^{2}$ of involved $p$ orbitals, i.e., of the $p$ character of the arrangement. The maximum strength $S_{m}=2.950$ is obtained with $a_{p}=3 / 4 \sqrt{2}$, corresponding to $s d^{15 / 8} p^{9 / 8}$ orbitals. Of these orbitals we write, for reason of brevity only $\psi_{1}$, which is

$$
\psi_{1}=\frac{1}{2} s+(3 / 4 \sqrt{2}) p_{z}+\frac{1}{4}(15 / 2)^{\frac{1}{2}} d_{z} .
$$

The five non-bonding orbitals, which are orthogonal to the tetrahedral orbitals given by (14a), (14b), (14c), (14d), (17), and (18), may be given by the following expressions :

$$
\begin{gathered}
\psi_{5}=\left(\frac{2}{3}\right)^{\frac{1}{2}} d_{y+z}-(1 / \sqrt{3}) d_{x+y} \\
\psi_{6}=\left(\frac{2}{3}\right)^{\frac{1}{2}} d_{x+z}+(1 / \sqrt{3}) d_{x y} \\
\psi_{7}=(2 / 3 \sqrt{3})\left[\left(\frac{3}{4}-a_{p}^{2}\right)^{\frac{1}{2}}\left(-p_{x}+2 p_{y}-2 p_{z}\right)\right. \\
+a_{p}\left(2 d_{z}+2\left\{\left(\frac{2}{3}\right)^{\frac{1}{2}} d_{x+y}+(1 / \sqrt{3}) d_{y+z}\right\}\right. \\
\left.\left.\quad-\left\{\left(\frac{2}{3}\right)^{\frac{1}{2}} d_{x y}-(1 / \sqrt{3}) d_{x+z}\right\}\right)\right], \\
\psi_{8}=(2 / 3 \sqrt{3})\left[\left(\frac{3}{4}-a_{p}^{2}\right)^{\frac{1}{3}}\left(2 p_{x}-p_{y}-2 p_{z}\right)\right. \\
+a_{p}\left(2 d_{z}-\left\{\left(\frac{2}{3}\right)^{\frac{1}{3}} d_{x+y}+(1 / \sqrt{3}) d_{y+z}\right\}\right. \\
\left.\left.\quad+2\left\{\left(\frac{2}{3}\right)^{\frac{1}{3}} d_{x y}-(1 / \sqrt{3}) d_{x+z}\right\}\right)\right], \\
\psi_{9}=(2 / 3 \sqrt{3})\left[\left(\frac{3}{4}-a_{p}^{2}\right)^{\frac{1}{2}}\left(2 p_{x}+2 p_{y}+p_{z}\right)\right. \\
+a_{p}\left(-d_{z}+2\left\{\left(\frac{2}{3}\right)^{\frac{1}{2}} d_{x+y}+(1 / \sqrt{3}) d_{y+z}\right\}\right. \\
\left.\left.+2\left\{\left(\frac{2}{3}\right)^{\frac{1}{3}} d_{x y}-(1 / \sqrt{3}) d_{x+z}\right\}\right)\right] .
\end{gathered}
$$

It is seen that only two of these orbitals are pure $d$ orbitals. Furthermore, using the theorem 
that the $p$ content of any orbital is given by the sum of the squares of the coefficients of $p_{x}, p_{y}$, and $p_{z}$, it follows from $(22 \mathrm{c}),(22 \mathrm{~d})$, and $(22 \mathrm{e})$ that each of the three $d p$ non-bonding orbitals is composed of $\left(1-(4 / 3) a_{p}{ }^{2}\right) p$ orbitals and $(4 / 3) a_{p}{ }^{2} d$ orbitals. In the case of the strongest tetrahedral orbitals $\left(a_{p}=3 / 4 \sqrt{2}\right)$ each of these three orthogonal $d p$ non-bond orbitals becomes $5 / 8 p$ and $3 / 8 d$ in character.

\section{c. Comparison of Suitability of the Different Possible Bond Orbital Arrangements}

In Sections $2 a$ and $2 b$ we were looking for arrangements of four spd bond orbitals containing exactly two $p$ functions and being at the same time orthogonal to four pure $s-d$ orbitals. Both of these conditions are fulfilled by square bond orbital arrangements, as found in Section 3a.

These conditions, however, cannot be fulfilled simultaneously by tetrahedral orbitals (Section $3 b)$. We can in that case fulfill the first condition by choosing $m=4 a_{p}{ }^{2}=2$, corresponding to a $s p^{2} d$ tetrahedral bond orbital arrangement. Then we see, however, from Section $3 \mathrm{~b}$ that there are two $d$ and three $d^{\frac{2}{3}} p^{\frac{1}{3}}$ orbitals orthogonal to the tetrahedral bond orbitals thus obtained (and not four orbitals without $p$ character), the second condition, therefore, being unsatisfied.

A consideration, the details of which will not be given here, of irregular (trigonal axis deformed) tetrahedral and tetragonal pyramidal bond orbital functions leads to the same result as in the case of a regular tetrahedral arrangement just discussed, namely, that the two conditions mentioned above cannot be fulfilled simultaneously. Thus the square arrangement is the only arrangement of four equal bond orbitals that can be used under the conditions of Sections $2 \mathrm{a}$ and $2 \mathrm{~b}$.

In Section 2c we were looking for the four strongest $s p d$ bond orbitals that fulfill the condition of orthogonality to four $s-d$ or four pure $d$ orbitals. On examination of the tetrahedral orbitals of Section $3 b$ it is seen that this condition is fulfilled only in the case $m=4 a_{p}{ }^{2}=3$, corre- sponding to $s p^{3}$ tetrahedral orbitals of strength 2.00 .

On the other hand, according to Section 3a, there can be constructed four square bond orbitals with strength 2.943 which fulfill the condition of being orthogonal to four $s-d$ orbitals, and four square bond orbitals of strength 2.694 which are orthogonal to four pure $d$ orbitals. The strengths of these square bond orbitals are both much larger than the value 2.00 of the possible tetrahedral arrangement; this circumstance makes the tetrahedral arrangement unfavorable.

A similar consideration concerning irregular tetrahedral and tetragonal pyramidal orbitals leads to the same conclusion as in the case of regular tetrahedral orbitals. The square bond orbitals are thus seen to be the strongest four equivalent orbitals that are possible under the conditions of Section 2.

The possibility of an arrangement of nonequivalent bond orbitals that are stronger than the bond orbitals in square arrangements remains to be considered. It is not probable, however, that such an arrangement is possible. ${ }^{11}$

\section{ACKNOWLEDGMENTS}

The author is greatful to Professor Linus Pauling for many helpful suggestions and discussions and for the kind hospitality that he extended to him during his stay in Pasadena. $\mathrm{He}$ also wishes to thank Dr. David P. Shoemaker for help in the preparation of the manuscript and to express his gratitude to the Swiss Foundation "Stiftung für Stipendien auf dem Gebiete der Chemie" for a fellowship.

\footnotetext{
11 This conclusion can be drawn from a treatment, the details of which are omitted, concerning the four best $s p d$ orbitals that can be arranged in such a way that the strength vectors are directed from the center toward the corners of a rhombus. Such a treatment has shown that all bond orbital arrangements having the symmetry under discussion contain exactly two $p$ orbitals and that the four bond orbitals are orthogonal to four $s-d$ and one $p$ orbital, just as in the limiting case of square bond orbitals. Furthermore, it can be shown that of all rhombus bond orbital arrangements of a given composition in $s, p$, and $d$, the square arrangement is the one for which the sum of the strengths (or the sum of the squares of the strengths) of the four bonding orbitals turns out to be a maximum. This circumstance makes the square arrangement favorable.
} 